\title{
Estimates of perturbation series for kernels
}

\author{
KrzyszTof Bogdan, TOMASz JAKUbOWSKi AND SEbastian Sydor
}

Abstract. We consider Schrödinger-type perturbations of integral kernels on space-time. Under suitable conditions on the first nontrivial term of the perturbation series, we prove that the perturbed kernel is locally in time comparable with the original kernel.

\section{Introduction}

Estimates of the Green function and heat kernel of Schrödinger operators $\Delta+q$ were studied, for example, in $[9,8,14,20]$. Local integral smallness of the function $q$, defined via Kato-type conditions [8,20], played an important role in these considerations. Similar Schrödinger-type operators based on the fractional Laplacian $\Delta^{\alpha / 2}$ were studied in [1,2,6] (see also [7]), with focus on comparability of the resulting Green functions. The heat kernel estimates for $\Delta^{\alpha / 2}+q$, in fact Schrödinger-type perturbations of general transition densities, were then studied in [3] under the following integral condition on $q$,

$$
\int_{s}^{t} \int_{X} p(s, x, u, z)|q(u, z)| p(u, z, t, y) \mathrm{d} z \mathrm{~d} u \leq[\eta+\beta(t-s)] p(s, x, t, y),
$$

where $p$ is a finite jointly measurable transition density, $\beta$ and $\eta$ are fixed nonnegative numbers, while times $s<t$ and states $x, y$ are arbitrary. Given (1), the following estimate was obtained in [3],

$$
\tilde{p}(s, x, t, y) \leq \frac{1}{1-\eta} \exp \left(\frac{\beta}{1-\eta}(t-s)\right) p(s, x, t, y),
$$

provided $\eta<1$. Here $\tilde{p}$ denotes the Schrödinger perturbation series defined by $p$ and $q$ (see below for details). The approach of [3] depends on nontrivial combinatorics of the perturbation series. Further combinatorial arguments were used in [12] to refine the above result by skipping the Chapman-Kolmogorov condition on $p$, relaxing the

Mathematics Subject Classification (2010): 47A55, 60J35, 47D08

Keywords: Forward kernel, Kato class, Space-time.

The research was partially supported by grants MNiSW N N201 397137, MNiSW N N201 422539, and ANR-09-BLAN-0084-01. 
assumptions on $q$, and strengthening the estimate, as in (25) below. Meanwhile, a more straightforward method was proposed in [13] for gradient perturbations of the transition density of the fractional Laplacian. It was suggested in [13] that the technique may be applied to Schrödinger perturbations to produce the main results of [12]. In the present paper we develop this observation and estimate Schrödinger-type perturbations of Markovian semigroups, potential kernels, and general forward integral kernels on space-time by rather singular functions $q$. We obtain local in time and global in space comparability of the original and perturbed kernels under suitable conditions on the first nontrivial term of the perturbation series. We say that $f, g \geq 0$ are comparable if a number $c>0$ exists such that $c^{-1} f \leq g \leq c f$.

We wish to mention a related paper [4] on the von Neumann series of general integral kernels with a certain transience-type property. Both papers were inspired by $[3,12]$, but their methods and results are different. In particular, the present estimates are more convenient and precise for forward kernels in continuous time perturbed by functions.

In what follows we will assume that $q$ is nonnegative, since the absolute value of the perturbation with signed $q$ is bounded by the perturbation with $|q|$, if finite. In this connection we also note that a discussion of the positive lower bound for signed perturbations of transition densities is given in [3].

Our main results are given in Sect. 3. Examples of applications and further comments are given in Sect. 4. In particular, we estimate the inverse kernel of Schrödinger perturbations of Weyl fractional derivatives on the real line.

\section{Preliminaries}

Following [10], we recall some basic properties of kernels.

DEFINITION 1. Let $(E, \mathcal{E})$ be a measurable space. A kernel on $E$ is a map $K$ from $E \times \mathcal{E}$ to $[0, \infty]$ with the following properties:

1. $x \mapsto K(x, A)$ is $\mathcal{E}$-measurable for all $A \in \mathcal{E}$,

2. $A \mapsto K(x, A)$ is countably additive for all $x \in E$.

Consider kernels $K$ and $L$ on $E$. The map

$$
(x, A) \mapsto \int_{E} K(x, \mathrm{~d} y) L(y, A)
$$

from $(E \times \mathcal{E})$ to $[0, \infty]$ is a kernel on $E$, called the composition of $K$ and $L$, and denoted $K L$. Composition of kernels is associative [10]. We write $q \in \mathcal{E}^{+}$if $q$ : $E \rightarrow[0, \infty]$ and $q$ is $\mathcal{E}$-measurable. We will denote by the same symbol the kernel $q(x, A)=q(x) 1_{A}(x)$. Here $1_{A}$ is the indicator function of $A$. We let $K_{n}=(K q)^{n} K$, $n=0,1, \ldots$. Associativity yields the following.

LEMMA 1. $K_{n}=K_{n-1-m} q K_{m}$ for all $n \in \mathbb{N}$ and $m=0,1, \ldots, n-1$.

We will consider the perturbation of $K$ by $q$, defined as the kernel 


$$
\tilde{K}=\sum_{n=0}^{\infty} K_{n}=\sum_{n=0}^{\infty}(K q)^{n} K
$$

Of course, $K \leq \tilde{K}$. In what follows we will prove upper bounds for $\tilde{K}$ under additional conditions on $K$ and $K_{1}=K q K$.

\section{Estimates for kernels on space-time}

In what follows we consider a set $X$ (the state space) with $\sigma$-algebra $\mathcal{M}$, the real line $\mathbb{R}$ (the time) equipped with the Borel sets $\mathcal{B}_{\mathbb{R}}$, and $E=\mathbb{R} \times X$ (the space-time) with the product $\sigma$-algebra $\mathcal{E}=\mathcal{B}_{\mathbb{R}} \times \mathcal{M}$. We also fix $q \in \mathcal{E}^{+}$, a number $\eta \in[0, \infty)$, and a function $Q: \mathbb{R} \times \mathbb{R} \rightarrow[0, \infty)$ satisfying the following condition of super-additivity:

$$
Q(u, r)+Q(r, v) \leq Q(u, v) \text { for all } u<r<v
$$

Let $K$ be a kernel on $E$. We will assume that $K$ is a forward kernel, that is,

$$
K(s, x, A)=0 \text { provided } A \subseteq(-\infty, s] \times X \quad(A \in \mathcal{E}, s \in \mathbb{R}) .
$$

REMARK 1. In the language of [4], $(s, \infty) \times X$ is absorbing for forward kernels. We will also assume that

$$
K q K(s, x, A) \leq \int_{A} K(s, x, \mathrm{~d} t \mathrm{~d} y)[\eta+Q(s, t)], \quad(s, x) \in E, A \in \mathcal{E} .
$$

From now on (6) and similar inequalities will be abbreviated as follows,

$$
K_{1}(s, x, \mathrm{~d} t \mathrm{~d} y) \leq K(s, x, \mathrm{~d} t \mathrm{~d} y)[\eta+Q(s, t)] .
$$

THEOREM 1. For all $n=1,2, \ldots$, and $(s, x) \in E$,

$$
\begin{aligned}
K_{n}(s, x, \mathrm{~d} t \mathrm{~d} y) & \leq K_{n-1}(s, x, \mathrm{~d} t \mathrm{~d} y)\left[\eta+\frac{Q(s, t)}{n}\right] \\
& \leq K(s, x, \mathrm{~d} t \mathrm{~d} y) \prod_{k=1}^{n}\left[\eta+\frac{Q(s, t)}{k}\right] .
\end{aligned}
$$

If $0<\eta<1$, then for all $(s, x) \in E$,

$$
\tilde{K}(s, x, \mathrm{~d} t \mathrm{~d} y) \leq K(s, x, \mathrm{~d} t \mathrm{~d} y)\left(\frac{1}{1-\eta}\right)^{1+Q(s, t) / \eta} .
$$

If $\eta=0$, then for all $(s, x) \in E$,

$$
\tilde{K}(s, x, \mathrm{~d} t \mathrm{~d} y) \leq K(s, x, \mathrm{~d} t \mathrm{~d} y) e^{Q(s, t)} .
$$

Proof. By (6), we get (8) for $n=1$. By induction, Lemma 1, (6) and (5), 


$$
\begin{aligned}
(n+1) K_{n+1}(s, x, A)= & n K_{n} q K(s, x, A)+K_{n-1} q K_{1}(s, x, A) \\
\leq & n \int_{E} K_{n-1}(s, x, \mathrm{~d} u \mathrm{~d} z)\left[\eta+\frac{Q(s, u)}{n}\right] q(u, z) K(u, z, A) \\
& +\int_{A} \int_{E} K_{n-1}(s, x, \mathrm{~d} u \mathrm{~d} z) q(u, z) K(u, z, \mathrm{~d} t \mathrm{~d} y)[\eta+Q(u, t)] \\
\leq & \int_{A} K_{n}(s, x, \mathrm{~d} t \mathrm{~d} y)[(n+1) \eta+Q(s, t)],
\end{aligned}
$$

as needed. Then, (9) follows from (8), (11) results from Taylor's expansion of the exponential function, and (10) follows from the Taylor series

$$
(1-\eta)^{-a}=\sum_{n=0}^{\infty} \frac{\eta^{n}(a)_{n}}{n !}
$$

where $0<\eta<1, a \in \mathbb{R}$, and $(a)_{n}=a(a+1) \cdots(a+n-1)$.

We note that the comparability in Theorem 1 is local in time as the factors in (10) and (11) are bounded if so are $s$ and $t$. The comparability is global in space, meaning that the factors are independent of $x$ and $\mathrm{d} y$.

Theorem 1 has two fine or pointwise variants, which we will state under suitable conditions. We fix a (nonnegative) $\sigma$-finite, nonatomic measure

$$
\mathrm{d} t=\mu(\mathrm{d} t)
$$

on $\left(\mathbb{R}, \mathcal{B}_{\mathbb{R}}\right)$ and a function $k(s, x, t, A)$ defined for $s<t, x \in X, A \in \mathcal{M}$, such that $(s, x, t) \mapsto k(s, x, t, A) \in[0, \infty)$ is jointly measurable. We will call $k$ a transition kernel if the following Chapman-Kolmogorov identity holds

$$
\int_{X} k(s, x, u, \mathrm{~d} z) k(u, z, t, A)=k(s, x, t, A), \quad s<u<t .
$$

For instance, if $p$ is a transition probability, and we let $k(s, x, t, A)=p_{s, t}(x, A)$, then $k$ is a transition kernel, provided it is jointly measurable. We let $k_{0}=k$, and for $n=1,2, \ldots$, we define

$$
k_{n}(s, x, t, A)=\int_{s}^{t} \int_{X} k_{n-1}(s, x, u, \mathrm{~d} z) q(u, z) k(u, z, t, A) \mathrm{d} u .
$$

LEMMA 2. If $n \in \mathbb{N}, m=0,1, \ldots, n-1, s<t, x \in X$ and $A \in \mathcal{E}$, then

$$
k_{n}(s, x, t, A)=\int_{s}^{t} \int_{X} k_{n-1-m}(s, x, u, \mathrm{~d} z) q(u, z) k_{m}(u, z, t, A) \mathrm{d} u .
$$

Proof. If $m=0$, then the equality (13) holds by the definition of $k_{n}$. In particular, this proves our claim for $n=1$. If $n \geq 1$ is such that (13) holds for all $m<n$, then so for every $m=1,2, \ldots, n$, we obtain 


$$
\begin{aligned}
k_{n+1}(s, x, t, A)= & \int_{s}^{t} \int_{X} k_{n}(s, x, u, \mathrm{~d} z) q(u, z) k(u, z, t, A) \mathrm{d} u \\
= & \int_{s}^{t} \int_{X} \int_{s}^{u} \int_{X} k_{n-1-(m-1)}\left(s, x, v, \mathrm{~d} z_{1}\right) q\left(v, z_{1}\right) k_{m-1}\left(v, z_{1}, u, \mathrm{~d} z\right) \mathrm{d} v \\
& \times q(u, z) k(u, z, t, A) \mathrm{d} u \\
= & \int_{s}^{t} \int_{X} k_{n-m}\left(s, x, v, \mathrm{~d} z_{1}\right) q\left(v, z_{1}\right) \\
& \times\left(\int_{v}^{t} \int_{X} k_{m-1}\left(v, z_{1}, u, \mathrm{~d} z\right) q(u, z) k(u, z, t, A) \mathrm{d} u\right) \mathrm{d} v \\
= & \int_{s}^{t} \int_{X} k_{n-m}\left(s, x, v, \mathrm{~d} z_{1}\right) q\left(v, z_{1}\right) k_{m}\left(v, z_{1}, t, A\right) \mathrm{d} v .
\end{aligned}
$$

We define

$$
\tilde{k}=\sum_{n=0}^{\infty} k_{n}
$$

We will assume that for all $s \leq t \in \mathbb{R}, x \in X$ and $A \in \mathcal{M}$,

$$
\int_{s}^{t} \int_{X} k(s, x, u, \mathrm{~d} z) q(u, z) k(u, z, t, A) \mathrm{d} u \leq[\eta+Q(s, t)] k(s, x, t, A),
$$

or $k_{1}(s, x, t, \mathrm{~d} y) \leq[\eta+Q(s, t)] k(s, x, t, \mathrm{~d} y)$. Thus, (15) is a fine version of (6).

THEOREM 2. For all $n=1,2, \ldots, s<t$ and $x \in X$,

$$
\begin{aligned}
k_{n}(s, x, t, \mathrm{~d} y) & \leq k_{n-1}(s, x, t, \mathrm{~d} y)\left[\eta+\frac{Q(s, t)}{n}\right], \\
& \leq k(s, x, t, \mathrm{~d} y) \prod_{k=1}^{n}\left[\eta+\frac{Q(s, t)}{k}\right] .
\end{aligned}
$$

If $0<\eta<1$, then for all $s<t$ and $x \in X$,

$$
\tilde{k}(s, x, t, \mathrm{~d} y) \leq k(s, x, t, \mathrm{~d} y)\left(\frac{1}{1-\eta}\right)^{1+Q(s, t) / \eta} .
$$

If $\eta=0$, then for all $s<t$ and $x \in X$,

$$
\tilde{k}(s, x, t, \mathrm{~d} y) \leq k(s, x, t, \mathrm{~d} y) e^{Q(s, t)} .
$$

Proof. By Lemma 2, induction, (15) and (4), for $n \geq 1$ we have

$$
\begin{aligned}
& (n+1) k_{n+1}(s, x, t, A) \\
& \quad \leq n \int_{s}^{t} \int_{X} k_{n-1}(s, x, u, \mathrm{~d} z)\left[\eta+\frac{Q(s, u)}{n}\right] q(u, z) k(u, z, t, A) \mathrm{d} u
\end{aligned}
$$




$$
\begin{aligned}
& +\int_{s}^{t} \int_{X} k_{n-1}(s, x, u, \mathrm{~d} z) q(u, z) k(u, z, t, A)\left[\eta+\frac{Q(u, t)}{n}\right] \mathrm{d} u \\
= & (n+1)\left[\eta+\frac{Q(s, t)}{n+1}\right] k_{n}(s, x, t, A), \quad A \in \mathcal{M} .
\end{aligned}
$$

For $n=1,(16)$ is identical with (15). We proceed as in Theorem 1.

For the finest variant of Theorem 1, we fix a $\sigma$-finite measure

$$
\mathrm{d} z=m(\mathrm{~d} z)
$$

on $(X, \mathcal{M})$.We will consider function $\kappa(s, x, t, y)$ defined for $s<t$ and $x, y \in X$, such that $(s, x, t, y) \mapsto \kappa(s, x, t, y) \in[0, \infty)$ is $\mathcal{B}_{\mathbb{R}} \times \mathcal{M} \times \mathcal{B}_{\mathbb{R}} \times \mathcal{M}$-measurable. We will call such $\kappa$ a (forward) kernel density, because $\int_{\{(t, y) \in E: s<t\}} \kappa(s, x, t, y) f(t, y) \mathrm{d} t \mathrm{~d} y$ is a forward kernel on $E$. For instance, we may take $k(s, x, t, y)=p_{s, t}(x, y)$, if measurable and finite, where $p$ is a transition probability density function. We define $\kappa_{0}(s, x, t, y)=\kappa(s, x, t, y)$,

$$
\kappa_{n}(s, x, t, y)=\int_{s}^{t} \int_{X} \kappa_{n-1}(s, x, u, z) q(u, z) \kappa(u, z, t, y) \mathrm{d} z \mathrm{~d} u, \quad n=1,2, \ldots
$$

LEMMA 3. For all $n=1,2, \ldots, m=0,1, \ldots, n-1, s, t \in \mathbb{R}$ and $x, y \in X$,

$$
\kappa_{n}(s, x, t, y)=\int_{s}^{t} \int_{X} \kappa_{n-1-m}(s, x, u, z) q(u, z) \kappa_{m}(u, z, t, y) \mathrm{d} z \mathrm{~d} u .
$$

Proof. The result was stated in [3, Lemma 3] under stronger conditions, so for the comfort of the reader we repeat the arguments of [3].

If $m=0$, then the equality (20) holds by the definition of $\kappa_{n}$. In particular, this proves our claim for $n=1$. If $n \geq 1$ is such that (20) holds for all $m<n$, then for every $m=1,2, \ldots, n$, by Fubini we indeed obtain

$$
\begin{aligned}
\kappa_{n+1}(s, x, t, y)= & \int_{s}^{t} \int_{X} \kappa_{n}(s, x, u, z) q(u, z) \kappa(u, z, t, y) \mathrm{d} z \mathrm{~d} u \\
= & \int_{s}^{t} \int_{X} \int_{s}^{u} \int_{X} \kappa_{n-1-(m-1)}\left(s, x, v, z_{1}\right) q\left(v, z_{1}\right) \kappa_{m-1}\left(v, z_{1}, u, z\right) \mathrm{d} z_{1} \mathrm{~d} v \\
& \times q(u, z) \kappa(u, z, t, y) \mathrm{d} z \mathrm{~d} u \\
= & \int_{s}^{t} \int_{X} \kappa_{n-m}\left(s, x, v, z_{1}\right) q\left(v, z_{1}\right) \\
& \times\left(\int_{v}^{t} \int_{X} \kappa_{m-1}\left(v, z_{1}, u, z\right) q(u, z) \kappa(u, z, t, y) \mathrm{d} z \mathrm{~d} u\right) \mathrm{d} z_{1} \mathrm{~d} v \\
= & \int_{s}^{t} \int_{X} \kappa_{n-m}\left(s, x, v, z_{1}\right) q\left(v, z_{1}\right) \kappa_{m}\left(v, z_{1}, t, y\right) \mathrm{d} z_{1} \mathrm{~d} v .
\end{aligned}
$$


The Schrödinger perturbation of $\kappa$ by $q$ is defined as follows:

$$
\tilde{\kappa}=\sum_{n=o}^{\infty} \kappa_{n}
$$

We will assume that for all $s<t \in \mathbb{R}$ and $x, y \in X$,

$$
\int_{s}^{t} \int_{X} \kappa(s, x, u, z) q(u, z) \kappa(u, z, t, y) \mathrm{d} z \mathrm{~d} u \leq[\eta+Q(s, t)] \kappa(s, x, t, y),
$$

or $\kappa_{1}(s, x, t, y) \leq \kappa(s, x, t, y)[\eta+Q(s, t)]$. This is a fine analogue of (6) and (15). The following is a fine version of Theorems 1 and 2 . We note that $(24,25,26)$, but not (23), were first proved in [12] by involved combinatorics.

THEOREM 3. For all $n=1,2, \ldots, s<t$ and $x, y \in X$,

$$
\begin{aligned}
\kappa_{n}(s, x, t, y) & \leq \kappa_{n-1}(s, x, t, y)\left[\eta+\frac{Q(s, t)}{n}\right] \\
& \leq \kappa(s, x, t, y) \prod_{k=1}^{n}\left[\eta+\frac{Q(s, t)}{k}\right] .
\end{aligned}
$$

If $0<\eta<1$, then for all $s<t$ and $x, y \in X$,

$$
\tilde{\kappa}(s, x, t, y) \leq \kappa(s, x, t, y)\left(\frac{1}{1-\eta}\right)^{1+Q(s, t) / \eta} .
$$

If $\eta=0$, then for all $s<t$ and $x, y \in X$,

$$
\tilde{\kappa}(s, x, t, y) \leq \kappa(s, x, t, y) e^{Q(s, t)} .
$$

Proof. We proceed as in the proof of Theorem 1, using Lemma 3 and (22).

\section{Discussion and applications}

The proofs of Theorems 1, 2, and 3 indicate that our estimates are rather tight. The observation is supported by the exact formulas for Schrödinger perturbations of transition densities by Dirac measures (not directly manageable by the methods of the present paper), see [4]. We like to note that the iterated integrals defining $K_{n}, k_{n}$, and $\kappa_{n}$ exhibit similarity to the expectations of powers of the additive functional in Khasminski's lemma [1,8], to Wiener chaoses and the multiple integrals in the theory of rough paths [15]. In fact, our results offer a far-reaching extension and strengthening of Khasminski's lemma for transition kernels and densities. On a formal level, a unique feature of our estimates is the combinatorics triggered by $\eta, Q$, and the assumptions (6), (15), (22). As we will see below, the room given by $\eta$ is quite convenient in applications, and $Q$ is often chosen linear.

In applications, we need to verify conditions (6), (15), or (22). 
EXAMPLE 1. Let $k(s, x, t, \mathrm{~d} y) \geq 0$ be a transition kernel, see (12). If $\mathrm{d} u$ is the linear Lebesgue measure and $\|q\|_{\infty}:=\sup |q(u, z)|<\infty$, then

$$
k_{1}(s, x, t, A) \leq\|q\|_{\infty} k(s, x, t, A) \int_{s}^{t} \mathrm{~d} u .
$$

Theorem 2, $Q(s, t)=\|q\|_{\infty}(t-s)$ and $\eta=0$ yield the well-expected bound,

$$
\tilde{k}(s, x, t, \mathrm{~d} y) \leq k(s, x, t, \mathrm{~d} y) e^{\|q\|_{\infty}(t-s)} .
$$

By Theorem 3, an analogous pointwise version of (27) also holds.

EXAMPLE 2. If $X=\left\{x_{0}\right\}$ consists of only one point and $\mathrm{d} z$ is the Dirac measure at $x_{0}$, then we can skip them from the notation. For instance, let $0<\beta<1, s<t$, and $\kappa(s, t)=\Gamma(\beta)^{-1}(t-s)^{\beta-1}$. For the linear Lebesgue measure $\mathrm{d} u$, Borel function $u \mapsto q(u) \geq 0$ and $s<t$,

$$
\begin{aligned}
\kappa_{1}(s, t) & =\frac{1}{\Gamma(\beta)^{2}} \int_{s}^{t}(u-s)^{\beta-1} q(u)(t-u)^{\beta-1} \mathrm{~d} u \\
& \leq \frac{\|q\|_{\infty}}{\Gamma(2 \beta)}(t-s)^{2 \beta-1}=\frac{\|q\|_{\infty}}{\Gamma(2 \beta)}(t-s)^{\beta} \kappa(s, t) \\
& \leq[\eta+c(t-s)] \kappa(s, t),
\end{aligned}
$$

provided $\|q\|_{\infty}<\infty$. Here $\eta>0$ may be arbitrarily small, at the expense of $c<\infty$. We note that such affine upper bounds are an important special case of (22), in particular (29) allows for an application of Theorem 3.

We can handle some unbounded functions $q$, too. For $s<u<t$ we have

$$
(u-s)^{1-\beta} \vee(t-u)^{1-\beta} \geq[(t-s) / 2]^{1-\beta},
$$

hence the following 3P Theorem holds for $\kappa$,

$$
\kappa(s, u) \wedge \kappa(u, t) \leq 2^{1-\beta} \kappa(s, t) .
$$

In consequence, $\kappa(s, u) \kappa(u, t) \leq 2^{1-\beta} \kappa(s, t)[\kappa(s, u)+\kappa(u, t)]$. By (28),

$$
\kappa_{1}(s, t) \leq \kappa(s, t) \frac{2^{1-\beta}}{\Gamma(\beta)}\left[\int_{s}^{t}(u-s)^{\beta-1} q(u) \mathrm{d} u+\int_{s}^{t}(t-u)^{\beta-1} q(u) \mathrm{d} u\right] .
$$

In particular, $q(u)=|u|^{-\beta+\varepsilon}$ with $0<\varepsilon \leq \beta$, yields sufficient smallness of the integrands in (30), hence local comparability of $\kappa$ and $\tilde{\kappa}$, by Theorem 3 .

REMARK 2. Let $\kappa$ be a (forward) kernel density. We will say that $q$ is of relative Kato class [3] for $\kappa$, if $\inf \left\{c: \int_{s}^{t} \int_{X} \kappa(s, x, u, z) q(u, z) \kappa(u, z, t, y) \mathrm{d} z \mathrm{~d} u \leq\right.$ $c \kappa(s, x, t, y)$ for all $\mathrm{s}<\mathrm{t}<\mathrm{s}+\mathrm{h}$ and $x, y \in X\} \rightarrow 0$ as $h \rightarrow 0$. In short,

$$
\sup \left\{\kappa_{1}(s, x, t, y) / \kappa(s, x, t, y): s<t<s+h, x, y \in X\right\} \rightarrow 0 \quad \text { as } h \rightarrow 0 .
$$


We say that $q$ is of Kato class for $\kappa$, if

$$
\sup \left\{\int_{s}^{t} \int_{X}[\kappa(s, x, u, z)+\kappa(u, z, t, y)] q(u, z) \mathrm{d} z \mathrm{~d} u\right\} \rightarrow 0 \text { as } h \rightarrow 0,
$$

where the supremum is taken over all $s<t<s+h$ and $x, y \in X$. The latter condition was proposed in [19] under the name of parabolic Kato condition, and the former was essentially used already in [20] to estimate Schrödinger perturbations of the Gaussian kernel. We note that the latter condition is usually weaker and easier to verify. As indicated by Example 2, when $\kappa$ satisfies the 3P Theorem, the Kato condition implies the relative Kato condition. Accordingly, the two are equivalent for the transition density of the fractional Laplacian $\Delta^{\alpha / 2}$ with $0<\alpha<2$, but not $\alpha=2$, because 3P fails for the Gaussian kernel. The details and further references are given in [3] for transition densities, see also [4] for the special case of Schrödinger perturbations of the Cauchy transition density.

We will make a connection to Schrödinger operators analogous to $\Delta+q$, as mentioned in Introduction. Consider a kernel $K$ on $E$, function $q \in \mathcal{E}^{+}$, and real-valued $\mathcal{E}$-measurable functions $\phi$ and $\psi$ on $E$ such that $K \psi=-\phi$. Here we assume absolute integrability: $K|\psi|<\infty$. Then,

$$
\begin{aligned}
\tilde{K}(\psi+q \phi) & =(K+\tilde{K} q K)(\psi+q \phi)=-\phi+K q \phi-\tilde{K} q \phi+\tilde{K} q K q \phi \\
& =-\phi+K q \phi-K q \phi-\tilde{K} q K q \phi+\tilde{K} q K q \phi=-\phi,
\end{aligned}
$$

provided the integrals are absolutely convergent for all arguments.

For forward kernels we can give rather explicit sufficient conditions for the absolute integrability. We will say $K$ is locally finite in time if for all real $s<t, u \in \mathbb{R}$ and $z \in X$, we have $K 1_{(s, t)}(u, z)=K(u, z,(s, t) \times X)<\infty$.

LEMMA 4. Consider a forward kernel $K$ locally finite in time. Let $q \in \mathcal{E}^{+}$satisfy (6) with $\eta<1$ and some superadditive function $Q$. Let $\psi$ and $\phi$ be real-valued $\mathcal{E}$ measurable functions such that $K \psi=-\phi$, and $|\psi| \leq c 1_{(a, b)}$ for some $a, b, c \in \mathbb{R}$. Then $\tilde{K}(\psi+q \phi)=-\phi$.

Proof. We have $|\phi| \leq K|\psi|<\infty$, by the local finiteness of $K$. By the preceding discussion it suffices to prove that $K q K|\psi|, \tilde{K} q K|\psi|$ and $\tilde{K} q K q K|\psi|$ are finite. In bounded time, by our assumptions and Theorem $1, K q K \leq C K, \tilde{K} \leq C K$, and $K q K q K \leq C K$, with some $C \in \mathbb{R}$, which ends the proof.

As a rule, if $K$ is a left inverse of an operator $L$ on space-time, then $\tilde{K}$ is a left inverse of $L+q$. Namely, if

$$
\int_{E} K(s, x, \mathrm{~d} u \mathrm{~d} z) L \phi(u, z)=-\phi(s, x), \quad(s, x) \in E,
$$

for some function $\phi$, then we consider $\psi=L \phi$ and obtain

$$
\int_{E} \tilde{K}(s, x, \mathrm{~d} u \mathrm{~d} z)[L \phi(u, z)+q(u, z) \phi(u, z)]=-\phi(s, x), \quad(s, x) \in E,
$$


under the assumptions of Lemma 4 . This is quite satisfactory if $L$ is local in time, because if $\phi$ is compactly supported in time, then so is $\psi$, and the boundedness of $\psi$ may usually be secured by appropriate assumptions on $\phi$, see, for example, $[3,5]$.

If $L$ is nonlocal in time, then more flexible conditions on $K$ may be needed.

LEMMA 5. Consider a forward kernel $K$ such that $K^{2}$ is locally finite in time. Let $q \in \mathcal{E}^{+}$satisfy (6) with $\eta<1$ and some superadditive function $Q$. Let $\psi$ and $\phi$ be real-valued $\mathcal{E}$-measurable functions such that $K \psi=-\phi$, and $|\psi| \leq c K 1_{(a, b)}$ for some $a, b, c \in \mathbb{R}$. Then $\tilde{K}(\psi+q \phi)=-\phi$.

Proof. The absolute integrability required for (31) amounts to the finiteness of $|\phi| \leq$ $K|\psi|, K q K|\psi|, \tilde{K} q K|\psi|$ and $\tilde{K} q K q K|\psi|$. In bounded time, by Theorem $1, K q K \leq$ $C K, \tilde{K} \leq C K$, and $K q K q K \leq C K$, with a number $C$. The result follows, since $K^{2} 1_{(a, b)}<\infty$ for finite $a<b$.

EXAMPLE 3. We consider the Weyl fractional integral on the real line [18],

$$
W^{-\beta} \psi(s)=\frac{1}{\Gamma(\beta)} \int_{s}^{\infty}(u-s)^{\beta-1} \psi(u) \mathrm{d} u .
$$

Here $\beta \in(0,1)$, and we require absolute integrability. The kernel has the density $\kappa(s, u)=(u-s)^{\beta-1} / \Gamma(\beta)$ discussed in Example 2. We also consider the Weyl fractional derivative,

$$
\partial^{\beta} \phi(s)=\frac{1}{\Gamma(1-\beta)} \int_{s}^{\infty}(u-s)^{-\beta} \phi^{\prime}(u) \mathrm{d} u .
$$

Here and in what follows $s \in \mathbb{R}$ and $\phi$ is a real-valued, continuously differentiable, and compactly supported function on $\mathbb{R}$. By Fubini's theorem,

$$
\begin{aligned}
W^{-\beta} \partial^{\beta} \phi(s) & =\frac{1}{\Gamma(\beta) \Gamma(1-\beta)} \int_{s}^{\infty} \int_{u}^{\infty}(u-s)^{\beta-1}(r-u)^{-\beta} \phi^{\prime}(r) \mathrm{d} r \mathrm{~d} u \\
& =\frac{1}{\Gamma(\beta) \Gamma(1-\beta)} \int_{s}^{\infty} \int_{s}^{r}(u-s)^{\beta-1}(r-u)^{-\beta} \phi^{\prime}(r) \mathrm{d} u \mathrm{~d} r \\
& =\int_{s}^{\infty} \phi^{\prime}(r) \mathrm{d} r=-\phi(s),
\end{aligned}
$$

see, for example, [18]. We intend to use Lemma 5. Let $\psi=\partial^{\beta} \phi$. If $a, b \in \mathbb{R}$ and $\operatorname{supp} \phi \subset(a, b)$, then $|\psi(s)| \leq(\Gamma(1-\beta))^{-1}\left\|\phi^{\prime}\right\|_{\infty} \int_{0}^{b-a} u^{-\beta} \mathrm{d} u$ for all $s \in \mathbb{R}$, and $\psi(s)=0$ for $s>b$. Since $\int_{a}^{b} \phi^{\prime}(u) \mathrm{d} u=0$, for $s<a$ we obtain

$$
\psi(s)=\frac{1}{\Gamma(1-\beta)} \int_{a}^{b}\left[(u-s)^{-\beta}-(a-s)^{-\beta}\right] \phi^{\prime}(u) \mathrm{d} u,
$$

hence $|\psi(s)| \leq(\Gamma(1-\beta))^{-1} \beta(b-a)^{2}(a-s)^{-\beta-1}\left\|\phi^{\prime}\right\|_{\infty}$. On the other hand,

$$
W^{-\beta} 1_{\left(a^{\prime}, b^{\prime}\right)}(s) \geq \frac{b^{\prime}-a^{\prime}}{\Gamma(\beta)}\left(b^{\prime}-s\right)^{\beta-1},
$$


if $s<a^{\prime}<b^{\prime}<\infty$. When multiplied by a constant, this majorizes $\psi$, provided $a^{\prime}>b$. Since $W^{-\beta} 1_{\left(a^{\prime}, b^{\prime}\right)}$ is locally bounded, and $W^{-\beta}$ is locally finite, we see that $\left(W^{-\beta}\right)^{2}$ is locally finite.

We now consider $q \in \mathcal{E}^{+}$satisfying (22) with $\eta<1$ and a superadditive function $Q$ (see Example 2 for such $q$ ). By Lemma 5 and the above discussion,

$$
\int_{s}^{\infty} \tilde{\kappa}(s, u)\left[\partial^{\beta} \phi(u)+q(u) \phi(u)\right] \mathrm{d} u=-\phi(s),
$$

where, by Theorem 3,

$$
\tilde{\kappa}(s, t)=\sum_{n=0}^{\infty} \kappa_{n}(s, t) \leq \frac{1}{\Gamma(\beta)}\left(\frac{1}{1-\eta}\right)^{1+Q(s, t) / \eta}(t-s)^{\beta-1}, \quad s<t .
$$

It seems that our methods also apply to perturbations of the so-called anomalous diffusions, which are driven by fractional time derivatives, see [11,16,17].

\section{Acknowledgments}

We thank Wolfhard Hansen and Karol Szczypkowski for useful comments.

Open Access. This article is distributed under the terms of the Creative Commons Attribution License which permits any use, distribution, and reproduction in any medium, provided the original author(s) and the source are credited.

\section{REFERENCES}

[1] Bogdan, K., and Byczkowski, T. Potential theory for the $\alpha$-stable Schrödinger operator on bounded Lipschitz domains. Studia Math. 133, 1 (1999), 53-92.

[2] Bogdan, K., and Byczkowski, T. Potential theory of Schrödinger operator based on fractional Laplacian. Probab. Math. Statist. 20, 2, Acta Univ. Wratislav. No. 2256 (2000), 293-335.

[3] Bogdan, K., Hansen, W., and Jakubowski, T. Time-dependent Schrödinger perturbations of transition densities. Studia Math. 189, 3 (2008), 235-254.

[4] Bogdan, K., Hansen, W., and Jakubowski, T. Localization and Schrödinger perturbations of kernels. Preprint (arXiv), 2012.

[5] Bogdan, K., and Jakubowski, T. Estimates of the Green function for the fractional Laplacian perturbed by gradient. Potential Anal. 36 (2012), 455-481.

[6] Chen, Z.-Q., and Song, R. Intrinsic ultracontractivity and conditional gauge for symmetric stable processes. J. Funct. Anal. 150, 1 (1997), 204-239.

[7] Chen, Z.-Q., and Song, R. General gauge and conditional gauge theorems. Ann. Probab. 30, 3 (2002), 1313-1339.

[8] Chung, K. L., and Zhao, Z. X. From Brownian motion to Schrödinger's equation, vol. 312 of Grundlehren der Mathematischen Wissenschaften [Fundamental Principles of Mathematical Sciences]. Springer-Verlag, Berlin, 1995.

[9] Cranston, M., Fabes, E., and Zhao, Z. Conditional gauge and potential theory for the Schrödinger operator. Trans. Amer. Math. Soc. 307, 1 (1988), 171-194.

[10] Dellacherie, C., and Meyer, P.-A. Probabilities and potential. C, vol. 151 of North-Holland Mathematics Studies. North-Holland Publishing Co., Amsterdam, 1988. Potential theory for discrete and continuous semigroups, Translated from the French by J. Norris. 
[11] Hahn, M., and Umarov, S. Fractional Fokker-Planck-Kolmogorov type equations and their associated stochastic differential equations. Fract. Calc. Appl. Anal. 14, 1 (2011), 56-79.

[12] Jakubowski, T. On combinatorics of Schrödinger perturbations. Potential Anal. 31, 1 (2009), 45-55.

[13] Jakubowski, T., and Szczypkowski, K. Time-dependent gradient perturbations of fractional Laplacian. J. Evol. Equ. 10, 2 (2010), 319-339.

[14] Liskevich, V., and Semenov, Y. Two-sided estimates of the heat kernel of the Schrödinger operator. Bull. London Math. Soc. 30, 6 (1998), 596-602.

[15] Lyons, T. J., Caruana, M., and Lévy, T. Differential equations driven by rough paths, vol. 1908 of Lecture Notes in Mathematics. Springer, Berlin, 2007. Lectures from the 34th Summer School on Probability Theory, Saint-Flour, July 6-24, 2004, with an introduction by J. Picard.

[16] Magdziarz, M. Stochastic representation of subdiffusion processes with time-dependent drift. Stochastic Process. Appl. 119, 10 (2009), 3238-3252.

[17] Meerschaert, M. M., Nane, E., and Vellaisamy, P. Distributed-order fractional diffusions on bounded domains. Journal of Mathematical Analysis and Applications 379, 1 (2011), 216-228.

[18] Miller, K. S., and Ross, B. An introduction to the fractional calculus and fractional differential equations. A Wiley-Interscience Publication. John Wiley \& Sons Inc., New York, 1993.

[19] Zhang, Q. On a parabolic equation with a singular lower order term. Trans. Amer. Math. Soc. 348, 7 (1996), 2811-2844.

[20] Zhang, Q. S. A sharp comparison result concerning Schrödinger heat kernels. Bull. London Math. Soc. 35, 4 (2003), 461-472.

\author{
Krzysztof Bogdan \\ Institute of Mathematics of the Polish \\ Academy of Sciences, \\ ul. Sniadeckich 8, 00-956 Warsaw, Poland \\ E-mail: bogdan@pwr.wroc.pl
}

Tomasz Jakubowski

Institute of Mathematics and Computer Science,

Wroctaw University of Technology,

Wybrzeże Wyspiańskiego 27, 50-370 Wrocław, Poland

E-mail:Tomasz.Jakubowski@pwr.wroc.pl

Sebastian Sydor

Institute of Mathematics,

University of Wroctaw,

pl. Grunwaldzki 2/4, 50-384 Wroctaw, Poland

E-mail: Sebastian.Sydor@math.uni.wroc.pl 